department. Co- created information by parents and health professionals were found to be more successful.

Conclusions Safety netting information resources may be more effective if they are designed with parents and their content, mode and place delivery is evidence based.

\section{IMPLEMENTING DEVELOPMENTAL SCREENING RECOMMENDATIONS IN A FAMILY MEDICINE RESIDENCY TRAINING PROGRAM: BARRIERS, SOLUTIONS AND RECOMMENDATIONS FOR PRACTICE}

doi:10.1136/archdischild-2012-302724.1750

'GA Devito, $2 \mathrm{~L}$ Manning, ${ }^{2} \mathrm{P}$ McLean, ${ }^{3} \mathrm{~A}$ Valeras. 'Department of Pediatrics Concord Hospital, New Hampshire Dartmouth Family Medicine Residency, Dartmouth Medical School; '2Family Health Center, NH Dartmouth Family Medicine Residency Program; ${ }^{3}$ Family Health Center, NH Dartmouth Family Medicine Residency, Dartmouth Medical School, Concord, NH, USA

Background and Aims The American Academy of Pediatrics recommends that all children, as part of well child care, have validated developmental screening in the medical home. Testing increases the early identification of children with developmental delay and improves outcome. However, USA data show that many primary care practices do not provide this screening. Our goal was to understand these barriers in our own practice, to overcome them, and to achieve $100 \%$ developmental screening rates.

Methods Our OI workgroup used electronic surveys and focus group discussion to solicit parent expectations, to understand provider barriers, and to explore work flow issues. Using this information to drive PDSA cycles and LEAN initiatives in our practice, we instituted educational training, workflow adjustments, screening tool modification, computerized tracking, and the redesign of select well child visits to focus on development screening.

Results The use of a validated screening tool by non-pediatric providers in our practice was $0 \%$ at the initiation of intervention. The major barriers to screening were lack of provider time, lack of comfort in developmental/behavioral pediatrics, lack of familiarity with a screening tool, and perceived lack of need for formalized testing. Following implementation of changes, including the modification of specific well visits to focus on developmental screening, $100 \%$ of eligible children had validated developmental screening performed over the last two months.

Conclusion Educational intervention, work flow modification, and the refocus of select well visits can significantly improve developmental screening rates in practice.

\section{CHILDPROOFING CANNOT BE SAFELY ASSUMED AFTER ACCIDENTAL POISON INGESTION RELATED HOSPITAL VISITS}

doi:10.1136/archdischild-2012-302724.1751

S Bharwani. Pediatrics, Faculty of Medicine and Health Sciences, UAE University, Al Ain, United Arab Emirates

Background/aims: Data is scant on the follow up of the patients after accidental poison ingestions. We wanted to determine if the parents of children with recent accidental poison ingestions took any steps for childproofing their homes after discharge from the hospital.

Method We conducted telephone interviews with the parents of children who visited ER (emergency center) of a tertiary center for accidental poison ingestions in the preceding 3-13 months. The parents were asked if they lock medicines in the cabinets, keep cleaning materials out of the reach of young children, educate their domestic helpers or do nothing to prevent such accidents. The parents had the option of selecting more than one strategy.
Results 100 parents were contacted and 71 decided to participate. 62 out of 71 households had a domestic helper but only 22/71 parents $(31 \%)$ said they would educate their domestic helpers. 39 parents $(55 \%)$ said they did or would lock the medicines in the cabinets and 35 out of $71(49 \%)$ said they would or did keep the cleaning materials out of reach of young children. Only 16 parents out of $71(22.5 \%)$ said they would do or did both- lock the medicines in the cabinet and keep the cleaning supplies out of reach of their young children.

Conclusion The domestic and behavior changes are not a foregone conclusion after accidental poison ingestions related ER visits. Follow up home visitation by a public health nurse for personalized tips in childproofing may have a positive behavioral outcome.

\section{A NATIONAL PROPOSAL IN COMMUNITY RISK PREVENTION: TRANSITION TO ADULT HEALTH CARE FOR ADOLESCENTS WITH CHRONIC DISEASES}

doi:10.1136/archdischild-2012-302724.1752

0 Urroz. National Cancer Institute Childrens Hospital, San Jose, Costa Rica

Childrens with complex chronic diseases now survive past the age of adolescence and into adult life, however health services have not always recognized the need to prepare these patients for the move into their specific adult services. This issue has resulted in these adolescents having to find a way to obtain the necessary health care services from adult healthcare providers However, without proper preparation on both ends of healthcare, this transition is very difficult. For all adolescents, the transition from childhood to adulthood involves combining the patient's individual identity, need to achieve independence, establishment of adult relationships and obtainment of a meaningful occupation. This transition is made more difficult because of the patient's continuous concerns about whether their social and health care needs will be met. These are the more serious reasons why many adolescents with chronic diseases are greatly hindered during the transition process. The effects of these issues are the debilitation and deterioration of the patient's health, both physical and psychological, mostly due to the fact that the patient is not prepared properly for the transition to adulthood with regards to their healthcare. The goal in this process is to make sure that the adolescent with chronic diseases, as well as their respective family members, are trained, helping to facilitate the ability to accept and understand that the transition process is like any other stage in life, with the inclusion of implication and new responsibilities specific to the patient that will need to be incorporated quickly and effectively.

\section{THE PERCEPTION OF THE TERM CEREBRAL PALSY (CP) IN SAUDI ARABIA}

doi:10.1136/archdischild-2012-302724.1753

S Madi, A Mandy, T Pountney. University of Brighton, Eastbourne, UK

Background and purposes: Cerebral palsy (CP) is one of the most common childhood disabilities and makes heavy demands on health, educational, and social services as well as on families and children. In Saudi Arabia the term CP is recognized by most health professionals as a physically disabling condition, however this is not the case for the general public, nor more importantly for mothers of children with CP. Misinterpreting the term of CP by Arabic language, clearly exists. This research aimed to explore the perception of the term CP with mothers of children with CP.

Methods Critical ethnography was adopted as the methodological approach; data were collected through focus groups, individual interviews, field note and participant observation. 